\title{
Prediction of Postoperative Visual Field Size from Preoperative Optic Chiasm Shape in Patients with Pituitary Adenoma
}

\author{
Sho Ito, ${ }^{*}$ Yuichiro YoneoKa, ${ }^{* *}$ Tetsuhisa Hatase, ${ }^{* * *}$ Yukihiko FuJII, ${ }^{* *}$ Takeo FukUChI, ${ }^{* * *}$ Atsuhiko IIJmaA ${ }^{*}, \#$
}

\begin{abstract}
Pituitary tumors frequently compress the optic chiasm, causing visual field deficits. Surgical removal of these tumors could improve visual functions. Thus, predicting the prognosis of visual field function is required, but currently there is no method for predicting postoperative visual field status from preoperative data of tumor and optic chiasm characteristics. In this study, we performed preoperative evaluation of visual field prognosis using numerical parameters in 40 patients with pituitary tumors. Data from 30 patients were used to calculate the regression equation and those from the remaining 10 patients were used to confirm the validity of these equations. We defined quantitative values (area of tumor, $A_{\text {tumor }}$; curvature of chiasm, $C_{\text {chiasm }}$; and area of chiasm, $A_{\text {chiasm }}$ ) based on tumor size, tumor shape, and optic chiasm shape as determined using magnetic resonance imaging. We determined pre- and postoperative visual field sizes by ophthalmologic methods, and quantified them as numerical values $(T N R)$. Postoperative recovery of the visual fields (obtained by comparing the post- and preoperative visual fields) was confirmed by increased postoperative TNRs $(P<0.01$ for 4 isopters and $P=0.01$ for 1 isopter, $t$-test). We attempted to predict postoperative visual field size using preoperative $A_{\text {tumor }}, C_{\text {chiasm }}$, and $A_{\text {chiasm }}$. Multiple regression analysis was performed, and three significant regression equations for predicting visual field size were obtained ( $\mathrm{N}=30, P<0.01, F$-test). The measured and predicted visual field sizes showed strong correlation $(\mathrm{N}=10, r>0.70)$. Thus, the quantitative parameters defined in this study clearly predicted postoperative visual functions in patients with pituitary tumor, and could find clinical applications in preoperative evaluation of visual field prognosis in neurosurgery.
\end{abstract}

Keywords: pituitary adenoma, visual field size, Goldmann perimeter, MRI, prognosis.

Adv Biomed Eng. 4: pp. 80-85, 2015.

\section{Introduction}

Pituitary tumors often compress the optic chiasm located above the pituitary organ, causing visual field deficits. Decompression of the optic chiasm by tumor removal results in visual field recovery [1-8]. Pituitary surgeons have tried to predict postoperative visual outcome using observations. Knowing the prognosis regarding visual fields would be very helpful to both clinicians and patients. Therefore, we determined whether the quantification of the tumor and optic chiasm characteristics could be used for preoperative evaluation of visual field prognosis.

Carrim et al. [9] reported linearity between the dimensions of the optic chiasm and visual fields investigated prior to surgical operations. Several previous studies have discussed the factors that affect postoperative visual fields [1-6] but were unable to predict postoperative visual fields using preoperative data. Yoneoka et al. [1] focused on the relationship between the visual field

This study was presented at the Symposium on Biomedical Engineering 2014, Tokyo, September, 2014.

Received on August 1, 2014; revised on October 26, 2014; accepted on December 19, 2014.

* Department of Bio-cybernetics, Graduate School of Science and Technology, Niigata University, Japan.

** Department of Neurosurgery, Brain Research Institute, Niigata University, Japan.

*** Division of Ophthalmology and Visual Science, Graduate School of Medical \& Dental Sciences, Niigata University, Japan.

\# 8050 2-Ikarashi, Nishi-ku, Niigata-shi, Niigata 950-2181, Japan. E-mail: a-iijima@eng.niigata-u.ac.jp and retinal thickness, and stated that early intervention would result in good postoperative visual field outcome. However, they did not estimate the postoperative visual field from preoperative information. Therefore, we focused on the relationship between visual field size and shape of the optic chiasm, and attempted to predict the postoperative visual field size from the preoperative optic chiasm shape.

In this work, we quantified the visual fields and characteristics of the optic chiasm, and evaluated these quantities by comparing their pre- and post-operative values. Further, we proposed a method of preoperative evaluation of visual field prognosis by predicting the postoperative visual field from the preoperative characteristics of the optic chiasm.

\section{Methods}

\subsection{Subjects}

We analyzed 40 subjects (male/female: $21 / 19$; age: $27-78$ years [mean \pm SD: $55.4 \pm 15.4]$ ), diagnosed with nonfunctioning pituitary adenoma. These subjects fulfilled the following inclusion criteria: (1) pressure on the visual pathway was symptomatic, (2) pituitary adenoma compressing the visual pathway was confirmed by magnetic resonance (MR) imaging, (3) patient underwent endoscopic endonasal transsphenoidal resection at our hospital, and (4) tumors were histologically diagnosed as non-inflammatory lesions. Exclusion criteria were: (i) any inflammatory lesion, (ii) pediatric population (under 15 years), and (iii) presence of symptomatic cataract or other ophthalmic diseases. We obtained approval from the Niigata University Medical Department Ethical Review Board for the use of MRI and visual field data. 


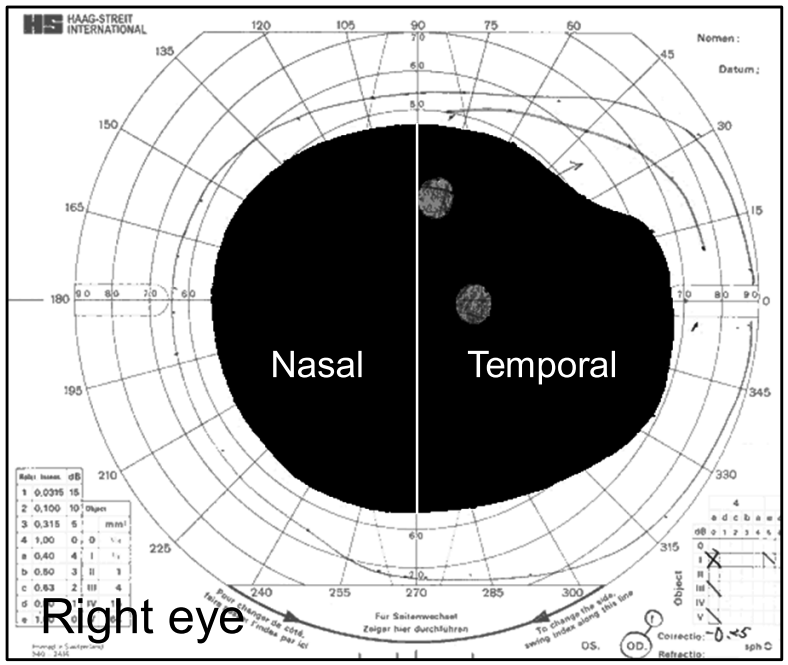

Fig. 1 Example of visual field (right eye, I/4e) measured by Goldmann perimeter. The area inside the isopter, excluding the scotoma, was calculated for the visual field. The ratio of the area of nasal to temporal visual field: TNR.

\subsection{Quantification of the visual field}

\subsubsection{Measuring the visual field}

While static perimeter is capable of automatic measurement, the measurement target is only the central visual field. Symptoms of the visual field in pituitary tumor cases involve prominently the peripheral visual field [3-9]. Therefore, experienced technicians measured the visual field with Goldmann kinetic perimetry (GP; L-1560, Inami, Japan), which can measure the peripheral visual field. Ophthalmological evaluations were performed within 2 weeks before treatment and 1-2 weeks after treatment.

\subsubsection{Parameter for the visual field: $T N R$}

We focused on I/1e, I/2e, I/3e, I/4e, and V/4e, which are typical isopters. We converted Goldmann visual field images to bitmap images, and quantified the visual field size by extracting the area of each isopter. GIMP (version 2.6.11) was used to edit the images. Areas of absolute scotomas were excluded from the area of visual field. The parts where the isopter was disconnected were interpolated with a straight line (Fig. 1).

Pituitary tumors compress the optic chiasm, which corresponds to the temporal visual fields. Hence, visual field defects occur bitemporally. Gnanalingham et al. [2] reported that the extent of visual field recovery is dependent on the preoperative temporal visual field deficit. Therefore, we defined TNR, calculated as the ratio of the area of the nasal visual field to that of the temporal visual field, as the visual field parameter (Eq. 1). We can observe changes of the temporal visual field using TNR.

$$
T N R=\frac{\text { Area of Temporal }[\text { pixel }]}{\text { Area of Nasal }[\text { pixel }]}
$$

Pituitary adenomas would affect both the left and right eye visual fields. We obtained the unified visual field by integrating the left and right eye visual fields, and investigated the relationship between the optic chiasm shape and visual field.
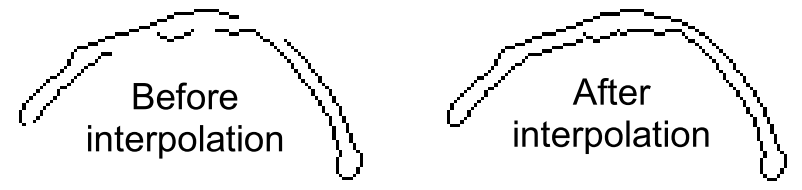

Fig. 2 Left: a contour of the optic chiasm selected by the clinician (step IV); right: reproducing contour after interpolation of the defects (step V).

\subsection{Quantification of optic chiasm shape \\ 2.3.1 Brain imaging}

Pituitary MR imaging at 3.0-T (Siemens, Germany) was performed before treatment and 1-2 weeks after treatment. Contact between the tumor and the visual pathway was assessed by preoperative MR imaging as an inclusion criterion.

\subsubsection{Extraction of chiasm edge}

We used coronal sections of the MR images that showed the longest lateral axis of the optic chiasm, because they most likely depict compression and extension by the tumor. These sections are considered to show the strongest correlation with visual field defects. A neurosurgeon independent of the analysts selected the MR images.

We performed MR image processing to quantify the optic chiasm shape, and extracted the contour of the optic chiasm. MATLAB (version 7.10.0) was used for image processing. The procedure of extraction was as follows: (I) conversion of MR image (PNG image) to grayscale, (II) contrast enhancement of the gray image, and (III) edge detection using the Canny method [10]. At this point, contour of the optic chiasm were divided into two groups:

0 . Outline is clear and separate

1. Contact with other parts of the brain

In case of 1 , (IV) selection of the contour of the optic chiasm was conducted under supervision of a clinician. In this case, contour defect occurs. Hence, (V) interpolation of the defect was conducted. The upper and under sides of the optic chiasm were approximated by a fifth-order function, and both ends of the defective portions were interpolated using quadratic functions (Fig. 2).

\subsubsection{Parameters for optic chiasm shape}

We defined 3 parameters for the optic chiasm shape:

1) Area of tumor $\left(A_{\text {tumor }}\right)$ : the dimensions of the optic chiasm compressed by the tumor. This indicates how the optic chiasm is shifted upwards (Fig. 3).

2) Curvature of chiasm $\left(C_{\text {chiasm }}\right)$ : flexion of the optic chiasm by calculating the $P$-type Fourier descriptor (Eq. 2).

$$
c(\mathrm{~s})=\frac{1}{N} \sum_{i=0}^{N-1} w_{i} e^{-j \frac{2 \pi}{N} s i}
$$

$\mathrm{s}$ : number of elements of the Fourier transform $0 \leq \mathrm{s} \leq N-1$

$i$ : number of elements of the complex sequence

$j:$ the imaginary unit

The P-type Fourier descriptor approximates the curve line using linear lines and angles [11]. Under the hypothesis that the shape of the normal optic chiasm is linear, we calculated the abnormality of the compressed optic chiasm as follows: First, we approximated the curve of the optic chiasm using the P-type Fou- 


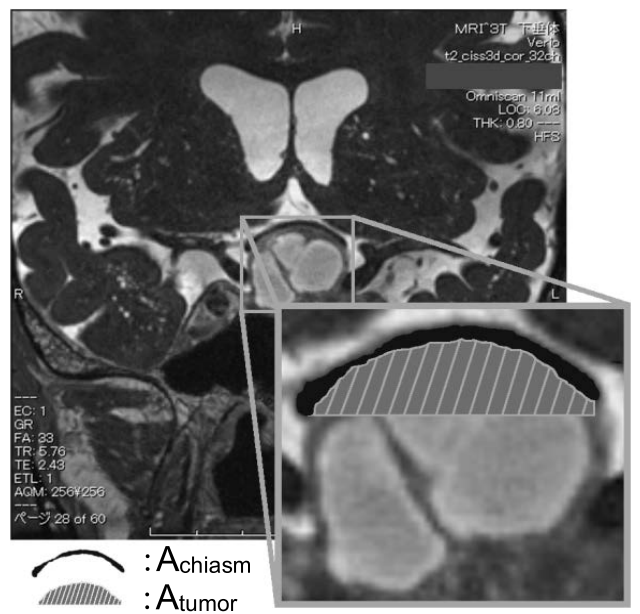

Fig. 3 A coronal MR image showing $A_{\text {tumor }}$ and $A_{\text {chiasm }}$.

rier descriptor. Next, a reference horizontal line was approximated using the same kind of parameters. Finally, we calculated the Euclidean distances between the approximated optic chiasm and reference line.

3) Area of chiasm $\left(A_{\text {chiasm }}\right)$ (Fig. 3).

\subsection{Prediction of postoperative visual field}

For the prediction of postoperative visual fields, we employed multiple regression analysis using the optic chiasm parameters $\left(A_{\text {tumor }}, A_{\text {chiasm }}, C_{\text {chiasm }}\right)$ as explanatory variables and the postoperative visual field as objective variable $[12,13]$. Prior to analysis, we confirmed multicollinearity among the explanatory variables by calculating the variance inflation factor (VIF) (Eq. 3).

$$
\begin{aligned}
& V I F_{j}=\frac{1}{1-R_{j}^{2}} \\
& j: \text { number of explanatory values } \\
& R: \text { multiple correlation coefficient }
\end{aligned}
$$

(1) $0<V I F<5$ : there is no evidence of multicollinearity, (2) $5 \leq V I F \leq 10$ : there is moderate multicollinearity, (3) $V I F>10$ : there is high multicollinearity in the variables.

After confirming no multicollinearity, we conducted multiple regression analysis to predict postoperative visual fields using data from 30 subjects randomly selected from our 40 participants. Furthermore, we evaluated the validity of the regression equations obtained using the data from the remaining 10 subjects which were not used for the prior regression analysis.

We used MATLAB (ver. 7.10.0) for all statistical analyses.

\section{Results}

\subsection{Evaluation of pre/postoperative $T N R$}

Figure 4 shows the scattergram of the pre/post-operative TNR. The $y=x$ line in the figure is a criterion; plots above the line indicate $T N R$ increase after surgery. The difference between pre- and postoperative $T N R$ on each isopter was significant (Table 1). Table 1 shows increases and decreases of TNR from before to after operation.

\subsection{Evaluation of pre/postoperative optic chiasm parame- ter}

Scattergrams of $A_{\text {tumor }}, C_{\text {chiasm }}$, and $A_{\text {chiasm }}$ are shown in Figs. 5 to $7(\mathrm{~N}=40)$. Plots in the area below the $y=x$ line indicate that de-

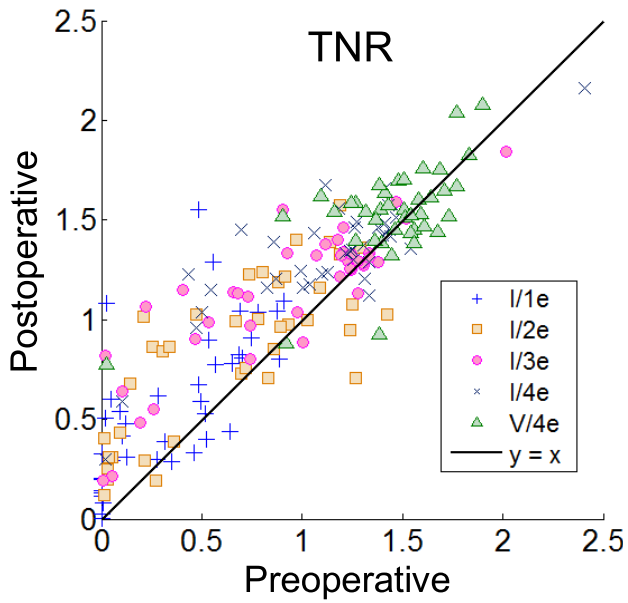

Fig. 4 Scattergram of pre/postoperative TNR. Line " $y=x$ " indicates the evaluation criterion: $T N R$ increase or decrease after operation.

Table 1 Results of paired $t$-test, and increases and decreases of TNR from before to after operation.

\begin{tabular}{cccccc}
\hline $\mathbf{N}=\mathbf{4 0}$ & $\mathbf{I} / \mathbf{1 e}$ & $\mathbf{I} / \mathbf{2 e}$ & $\mathbf{I} / \mathbf{3 e}$ & $\mathbf{I} / \mathbf{4 e}$ & $\mathbf{V} / \mathbf{4 e}$ \\
\hline $\boldsymbol{P}$ value & $<0.01$ & $<0.01$ & $<0.01$ & $<0.01$ & 0.01 \\
increase & 35 & 32 & 32 & 34 & 23 \\
decrease & 5 & 8 & 8 & 6 & 17 \\
\hline
\end{tabular}

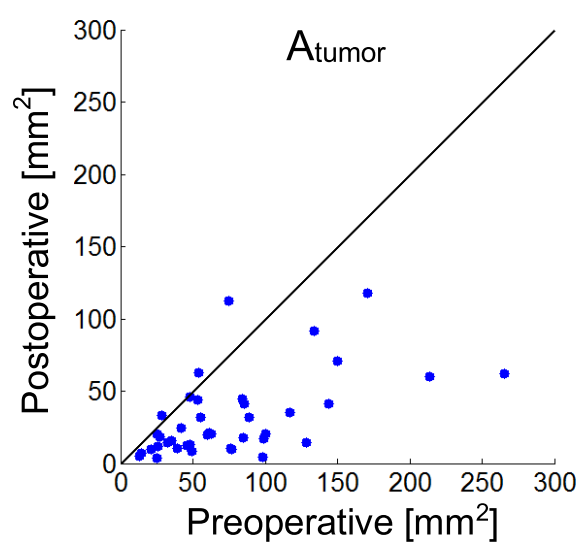

Fig. 5 Scattergram of pre/post-operative $A_{\text {tumor }}$. Difference between pre- and post-operative $A_{\text {tumor }}$ is significant $(P<0.01$, paired $t$-test).

crease of the parameter after surgery. The differences in pre- and postoperative parameters were significant for $A_{\text {tumor }}$ and $C_{\text {chiasm }}$ ( $P<0.01$, paired $t$-test $)$ but not for $A_{\text {chiasm }}(P=0.36$, paired $t$-test $)$.

\subsection{Multiple regression analysis}

Using data from 30 of the 40 subjects, we obtained equations to predict postoperative visual fields. Firstly, we confirmed multicollinearity among the three explanatory valuables $\left(A_{\text {tumor }}, C_{\text {chiasm }}\right.$, and $A_{\text {chiasm }}$ ) by VIF calculations. The results of VIF analysis are shown in Table 2. All VIF ranged from 0 to 5, and there was no multicollinearity. Therefore, we used the three parameters for multiple regression analysis. 


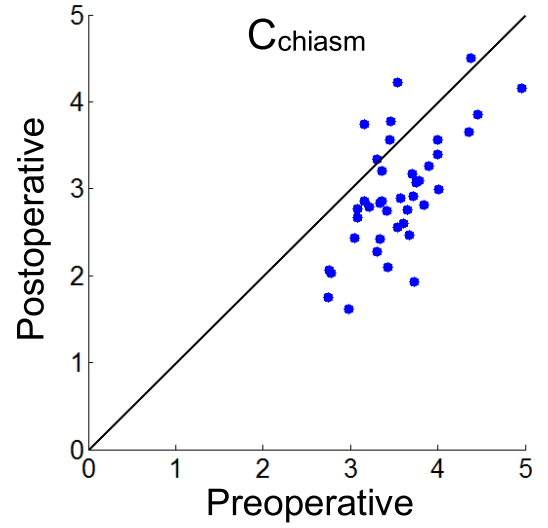

Fig. 6 Scattergram of pre/postoperative $C_{\text {chiasm }}$. Difference between pre- and post-operative $C_{\text {chiasm }}$ is significant $(P<0.01$, paired $t$-test).

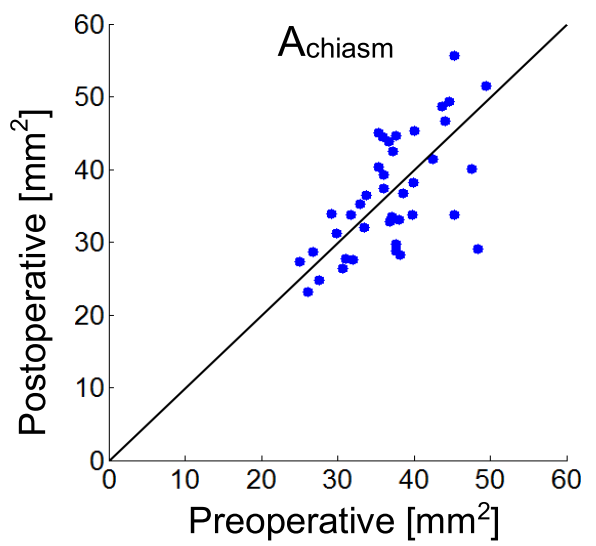

Fig. 7 Scattergram of pre/postoperative $A_{\text {chiasm }}$. Difference between pre- and post-operative $A_{\text {chiasm }}$ is not significant $(P=0.36$, paired $t$-test)

Table 2 Results of VIF analysis.

\begin{tabular}{cccc}
\hline $\mathbf{N}=\mathbf{3 0}$ & $\boldsymbol{A}_{\text {tumor }}$ & $\boldsymbol{C}_{\text {chiasm }}$ & $\boldsymbol{A}_{\text {chiasm }}$ \\
\hline $\boldsymbol{V I F}$ & 3.3 & 3.5 & 1.2 \\
\hline
\end{tabular}

Next, we predicted postoperative visual field (post-TNR) by multiple regression analysis, using preoperative $A_{\text {tumor }}, C_{\text {chiasm }}$, and $A_{\text {chiasm }}$ as explanatory values $(\mathrm{N}=30)$. The equations were significant for $\mathrm{I} / 1 \mathrm{e}, \mathrm{I} / 2 \mathrm{e}$, and $\mathrm{I} / 3 \mathrm{e}(P<0.01 ; F$-test $)($ Eqs. $4-6)$, and not significant for $\mathrm{I} / 4 \mathrm{e}(P=0.07)$ and $\mathrm{V} / 4(P=0.65)$. Significant equations are as follows:

$$
\begin{aligned}
I / 1 e= & 0.20-0.007 \times A_{\text {tumor }}+0.388 \times C_{\text {chiasm }} \\
& -0.012 \times A_{\text {chiasm }} \\
I / 2 e= & -0.01-0.008 \times A_{\text {tumor }} \\
& +0.346 \times C_{\text {chiasm }}+0.007 \times A_{\text {chiasm }} \\
I / 3 e= & 1.09-0.005 \times A_{\text {tumor }}-0.036 \times C_{\text {chiasm }} \\
& +0.008 \times A_{\text {chiasm }}
\end{aligned}
$$

Furthermore, we evaluated the validity of the above equations using the data of 10 subjects, which were not used for the above regression analysis. The parameters $A_{\text {tumor }}, C_{\text {chiasm }}$, and $A_{\text {chiasm }}$ from the data of the 10 subjects were plugged into equa-
Table 3 Correlation coefficients between measured and predicted values $(\mathrm{N}=10)$.

\begin{tabular}{cccc}
\hline TNR & I/1e & I/2e & I/3e \\
\hline $\boldsymbol{r}$ & 0.73 & 0.86 & 0.82 \\
\hline
\end{tabular}

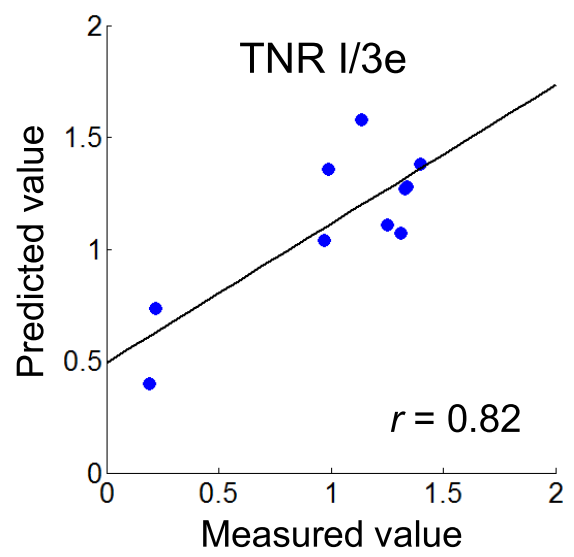

Fig. 8 Scattergram of measured and predicted postoperative TNR $\mathrm{I} / 3 \mathrm{e}(\mathrm{N}=10)$. The line was drawn by the least squares method. The correlation coefficient between the measured and predicted value was 0.82 , showing a strong correlation.

tions 4-6 to obtain the predicted visual field sizes. Table 3 shows the correlation coefficients $(r)$ between measured and predicted visual fields. The coefficients indicate strong correlation $(\mathrm{N}=10$, $r>0.70)$.

Figure 8 shows the scattergram between predicted and measured I/3e after operation.

\section{Discussion}

\subsection{Evaluation of pre/postoperative visual field}

On comparing pre- and post-operative $T N R$, we observed increased visual fields in all isopters. Thus, surgery likely decompressed the optic chiasm and improved visual fields. The increases in TNR indicate that recovery of the temporal visual field was better than that of the nasal visual field. This could be explained anatomically; the center of the optic chiasm includes the fibers for the temporal visual fields. This part is primarily compressed by pituitary tumors just below the chiasm [3-9]. Therefore, the pituitary tumors probably affect the temporal rather than the nasal visual field, and surgical intervention could significantly affect the temporal visual field.

However, we did also observe decreased postoperative TNR in some subjects. This could have been caused by optic nerve dysfunction due to prolonged compression by the tumor [1]. Further investigation involving measurement of the thickness of the retinal nerve fiber layer (RNFL) would be necessary in such cases. The decreased postoperative TNR could also be caused by the tumor compressing the off-center of the optic chiasm.

\subsection{Evaluation of pre/postoperative optic chiasm shape}

The changes in $A_{\text {tumor }}$ and $C_{\text {chiasm }}$ indicate that the compressed optic chiasm with a curved shape could transform to a normal linear shape. 
$A_{\text {chiasm }}$, which represents the area of the optic chiasm, did not change before and after the surgery. Thus, the optic chiasm is probably flexible, and stretching of the optic chiasm by the tumor could be reversed isovolumetrically.

These numerical observations throughout the parameters corresponded to the visual observations by the clinicians. Thus, our proposed parameters may accurately describe the correlation between the tumors and optic chiasm.

\subsection{Multiple regression analysis}

From the results of multiple regression analysis, the regression expressions for predicting I/1e to I/3e visual fields were significant. In the validity check of the equations, the correlation coefficients showed strong correlation between the measured and predicted visual fields. This result indicates that the proposed parameters are significantly related to the visual field function. The proposed method is potentially useful to evaluate the prognosis of patients who fulfilled the inclusion criteria with non-functioning pituitary tumors, based on the morphofunctional aspects of the visual system.

In the proposed methods, we had no information on the growth profiles of the adenoma. While the postoperative time course of the biological variation is easily detectable [2], it is quite difficult to know the history of the adenomas because each subject visited the hospital at different times. Even in the absence of pre-admission information about the course of tumor growth, our prediction values correlated highly with the measured values. In the next study, more precise prognosis prediction methods using temporal information will be developed. Furthermore, we will investigate the relationship between retinal thickness [1, 3] and the proposed parameters in detail. As previously reported on the retinal ganglion cell complex thickness in patients with stroke [14], the proposed method may be useful when combined with retinal information for the diagnosis of certain neurological diseases with visual function deficits.

\section{Conclusion}

The quantitative parameters of the visual field and optic chiasm shape defined in this study clearly predicted postoperative visual functions in patients with nonfunctioning pituitary tumor. Preoperative prognosis evaluation using such numerical indicators from the morphological aspects of the tumors and optic chiasm would be of great advantage in clinical neurosurgery. Furthermore, the proposed methods with some other neurological information may be useful for the diagnosis of diseases with impairment in visual field.

\section{Conflict of Interest}

We have no conflicts of interest or relationship with any companies or commercial organizations as defined by the Japanese Society of Medical and Biological Engineering.

\section{Dedication}

In memory of Dr. Mineo TAKAGI.

\section{References}

1. Yoneoka Y, Hatase T, Watanabe N, Jinguji S, Okada M, Takagi M, Fujii Y: Early morphological recovery of the optic chiasm is associated with excellent visual outcome in patients with com- pressive chiasmal syndrome caused by pituitary tumors. Neurol Res, in press, 2014.

2. Gnanalingham K, Bhattacharjee S, Pennington R, Ng J, Mendoza $\mathrm{N}$ : The time course of visual field recovery following transphenoidal surgery for pituitary adenomas: predictive factors for a good outcome. J Neurol Neurosurg Psychiatry. 76, pp. 415-419, 2005.

3. Garcia T, Sanchez S, Litré CF, Radoi C, Delemer B, Rousseaux P, Ducasse A, Arndt C: Prognostic value of retinal nerve fiber layer thickness for postoperative peripheral visual field recovery in optic chiasm compression. J Neurosurg. 121(1), pp. 165-169, 2014.

4. Findlay G, McFadzean RM, Teasdale G: Recovery of vision following treatment of pituitary tumors; Application of a new system of assessment to patients treated by transsphenoidal operation, Acta Neurochir (Wien). 68, pp. 175-186, 1983.

5. Barzaghi LR, Medone M, Losa M, Bianchi S, Giovanelli M, Mortini P: Prognostic factors of visual improvement after trans-sphenoidal approach for pituitary macroadenomas: review of the literature and analysis by quantitative method. Neurosurg Rev. 35(3), pp. 369-378, 2012.

6. Hudson H, Rissell C, Gauderman WJ, Feldon SE: Pituitary tumor volume as a predictor of postoperative visual field recovery: Quantitative analysis using automated static perimetry and computed tomography morphometry. J Clin Neurophthalmol. 11(4), pp. 280-283, 1991.

7. Laws ER Jr, Trautmann JC, Hollenhorst RW Jr: Transsphenoidal decompression of the optic nerve and chiasm. J Neurosurg. 46, pp. 717-722, 1977.

8. Svien L, Love JG, Kennedy WC, Colby MY Jr, Kearns TP: Status of vision following surgical treatment for pituitary chromophobe adenoma. J Neurosurg. 22, pp. 47-52, 1965.

9. Carrim ZI, Reeks GA, Chohan AW, Dunn LT, Hadley DM: Predicting impairment of central vision from dimensions of the optic chiasm in patients with pituitary adenoma. Acta Neurochir (Wien). 149, pp. 255-260, 2007.

10. Canny J: A computational approach to edge detection. IEEE Trance Pattern Anal Mach Intell. 8(6), pp. 679-698, 1986.

11. Yamada T, Ichihara K, Mori K, Saito T: A kinematics analysis of cervical vertebrae using X-ray projection images. Jpn J Clin Biomech. 32, pp. 141-148, 2011.

12. Hossain MG, Saw A, Alam R, Ohtsuki F, Kamarul T: Multiple regression analysis of anthropometric measurements influencing the cephalic index of male Japanese university student. Singapore Med J. 54(9), pp. 516-520, 2013.

13. Chatterjee S, Price B: Regression Analysis by Example, Second Edition. Wiley-Interscience, pp. 186-193, 1991.

14. Yamashita T, Miki A, Iguchi Y, Kimura K, Maeda F, Kiryu J: Reduced retinal ganglion cell complex thickness in patients with posterior cerebral artery infarction detected using spectral-domain optical coherence tomography. Jpn J Ophthalmol. 56(5), pp. 502-510, 2012.

\section{Sho Iто}

Mr. Sho ITo received the BS degree in Engineering from Niigata University in 2013. He is currently a graduate student of the Graduate School of Science and Technology, Niigata University. His research fields includes the analysis of MR imaging and ophthalmologic information.

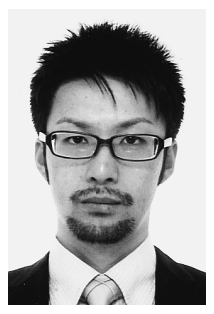




\section{Yuichiro YoNeOKA}

Dr. Yuichiro Yoneoka graduated from Kanazawa University in 1993 and received his $\mathrm{PhD}$ from the Graduate School of Medicine of Niigata University in 1999. He has been an Assistant Professor at the Niigata University Medical \& Dental Hospital (Neurosurgery) since 2007. He is a specialist in endoscopic surgery for hypothalamic and pituitary tumors.

\section{Tetsuhisa Hatase}

Dr. Tetsuhisa HaTASE received his medical degree in 2002 and his $\mathrm{PhD}$ in 2010 from Niigata University of Medicine, Niigata, Japan. He is a specialist in neuroophtalmology.

\section{Yukihiko FuJII}

Dr. Yukihiko FuJII graduated from Niigata University in 1983 and received his $\mathrm{PhD}$ in 1992. He has been the Chair of the Department of Neurosurgery since 2006 and an Assistant Director of the Niigata University Medical \& Dental Hospital since 2013. He has doubled as a Professor of the Department of Digital Medicine, Department of Neurosurgery, since 2006 . He is a specialist in functional neuroimaging.

\section{Takeo FuKUCHI}

Dr. Takeo FukuchI received his medical degree in 1985 and his $\mathrm{PhD}$ in 1991 from Niigata University of Medicine, Niigata, Japan. He is a Professor and Chair of the Division of Ophthalmology and Visual Science, Niigata University, Graduate School of Medical and Dental Sciences.

\section{Atsuhiko IıJIMA}

Dr. Atsuhiko IJImA received the BS, MS, and PhD degrees in Biomedical Engineering from Keio University in 1999, 2001, and 2003, respectively. He was a Research Fellow for the Young Scientists of the Japan Society for the Promotion of Science from 2002 to 2004. He was an Assistant Professor of the Graduate School of Medical \& Dental Sciences (Physiology), Niigata University, from 2004 to 2010. He is currently an Associate Professor in the Department of Bio-cybernetics, Faculty of Engineering, Niigata University. His research fields are neurophysiology and biomedical engineering in visual neurosciences. 